\title{
Social balance on networks: The dynamics of friendship and enmity
}

\author{
T. Antal, P.L. Krapivsky, S. Redner* \\ Center for Polymer Studies and Department of Physics, Boston University, Boston, MA 02215, United States
}

Available online 7 November 2006

\begin{abstract}
How do social networks evolve when both friendly and unfriendly relations exist? Here we propose a simple dynamics for social networks in which the sense of a relationship can change so as to eliminate imbalanced triads — relationship triangles that contains 1 or 3 unfriendly links. In this dynamics, a friendly link changes to unfriendly or vice versa in an imbalanced triad to make the triad balanced. Such networks undergo a dynamic phase transition from a steady state to "utopia" - all friendly links - as the amount of network friendliness, defined as the fraction of friendly links $\rho$, is changed. Basic features of the long-time dynamics and the phase transition are discussed.
\end{abstract}

(C) 2006 Elsevier B.V. All rights reserved.

Keywords: Social balance; Networks

\section{Introduction}

As we all have experienced, social networks can evolve in convoluted ways. Friendships can become estrangements and vice versa. New friendships can be created while existing friends drift apart. How are these changing relations reflected in the structure of social networks? As a familiar and illustrative example, suppose that you are friendly with a married couple that gets divorced. A dilemma arises if you try to remain friendly with both of the former spouses. You may find yourself in the uncomfortable position of listening to each of the former spouses separately disparaging each other. Ultimately you may find it simplest to remain friends with only one of the former spouses and to cut relations with the other ex-spouse. In the language of social balance [1-4], the initially balanced triad became unbalanced when the couple divorced. When you subsequently kept your friendship with only one former spouse, social balance is restored.

What happens in a larger social network? Now we need to look at all triads $i j k$ that link individuals $i, j$, and $k$. We define the link variable $s_{i j}=1$ if $i$ and $j$ friends and $s_{i j}=-1$ otherwise. Then the triad $i j k$ is balanced if $s_{i j} s_{j k} s_{k i}=1$, and is imbalanced otherwise (Fig. 1). A balanced triad therefore fulfills the adage:

\footnotetext{
* Corresponding author. Tel.: +1 617 3532618; fax: +1 6173539393 .

E-mail address: redner@bu.edu (S. Redner).
}

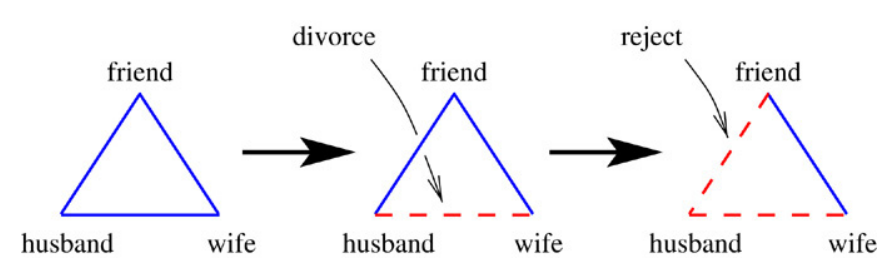

Fig. 1. Evolution of a married couple plus friend triad. After a divorce the triad becomes imbalanced, but balance is restored after another relationship changes. Full and dashed lines represent friendly and unfriendly relations respectively.

- a friend of my friend, as well as an enemy of my enemy, is my friend;

- a friend of my enemy, as well as an enemy of my friend, is my enemy.

A network is balanced if each constituent triad is balanced $[1,4]$. A seemingly more general definition of a balanced network is to require that each closed cycle is balanced; that is, $\prod_{\ell \in \text { path }} s_{\ell}=+1$. Cartwright and Harary showed [5] that a cycle-based definition of balance is equivalent to a triad-based definition for complete graphs. This result can be reformulated as follows: if we detect an imbalanced cycle of any length in a complete graph, there must be an imbalanced triad.

Balance theory was originally introduced by Heider [1] and important contributions were made by many others $[2,3,6]$. Cartwright and Harary [5,7] translated Heider's ideas into the framework of graph theory, and proved several fundamental theorems about the structure of balanced networks. There is 
also an extensive literature on balance theory (see e.g., $[4,8-13]$ and references therein).

Cartwright and Harary [5] showed that on a complete graph balanced societies are remarkably simple: either all individuals are mutual friends ("utopia"), or the network segregates into two mutually antagonistic but internally friendly cliques a "bipolar" state (see Fig. 10 as an example). However, spontaneously balanced states are rare - if one were to assign relationships in a social network at random, the probability that this society is balanced would vanish exponentially with system size. Thus to understand how a network reaches a balanced state we need to go beyond static descriptions to investigate how an initially imbalanced society becomes balanced via social dynamics.

Here we discuss the evolution of such social networks when we allow the sense of each link to change from friendly to unfriendly or vice versa to reflect the natural human tendency to reduce imbalanced triads [14,15]. Two such dynamics are considered: local triad dynamics (LTD) and constrained triad dynamics (CTD). For simplicity, we consider complete graph networks - everyone knows everyone else. We will address the basic question: what is the long-time state of such networks?

\section{Local triad dynamics}

\subsection{The update rule}

In local triad dynamics (LTD), an imbalanced triad is selected at random and the sign of a relationship between two individuals is flipped to restore the triad to balance. This change is made irregardless if other triads become imbalanced as a result. Thus LTD can be viewed as the social graces of the clueless - such a person makes a relationship change without considering the ramifications on the rest of his social network. We define a triad $\Delta$ to be of type $k$ if it contains $k$ unfriendly links. Thus $\triangle_{0}$ and $\Delta_{2}$ are balanced, while $\Delta_{1}$ and $\Delta_{3}$ are imbalanced. With these definitions, the LTD rules are (Fig. 2):

(1) Pick a random triad; if it is balanced do nothing.

(2) If the triad is of type $\triangle_{1}$, then: (i) with probability $p$, change the unfriendly link to a friendly link; (ii) with probability $1-p$, change a friendly link to an unfriendly link.

(3) If the triad is of type $\Delta_{3}$, then change an unfriendly link to a friendly link.

After the update, the initial imbalanced target triad becomes balanced, but other previously-balanced triads that share a link with this target may become imbalanced. These triads can subsequently evolve and return to balance, leading to new imbalanced triads. For example, when a married couple breaks up, friends of the former couple that remain friends with the former wife may then redefine their relationships with those who choose to remain friends with the former husband. These redefinitions, may lead to additional relationship shifts, etc.
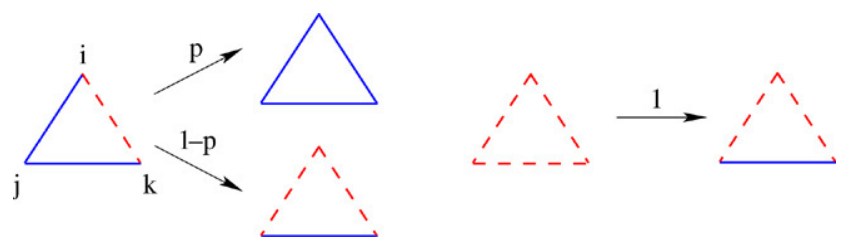

Fig. 2. An update step on imbalanced triads $\Delta_{1}$ (left) and $\triangle_{3}$ (right) by local triad dynamics. Solid and dashed lines represent friendly and unfriendly links, respectively.

\subsection{Evolution on the complete graph}

We now study LTD on a finite complete graph of $N$ nodes, $L=\left(\begin{array}{c}N \\ 2\end{array}\right)$ links, and $N_{\triangle}=\left(\begin{array}{c}N \\ 3\end{array}\right)$ triads. Let $N_{k}$ be the number of triads that contain $k$ unfriendly links, with $n_{k}=N_{k} / N_{\triangle}$ the respective triad densities, and $L^{+}\left(L^{-}\right)$the number of friendly (unfriendly) links. The number of triads and links are related by

$L^{+}=\frac{3 N_{0}+2 N_{1}+N_{2}}{N-2}, \quad L^{-}=\frac{N_{1}+2 N_{2}+3 N_{3}}{N-2}$.

The numerator counts the number of friendly links in all triads while the denominator appears because each link is counted $N-2$ times. The density of friendly links is therefore $\rho=L^{+} / L=\left(3 n_{0}+2 n_{1}+n_{2}\right) / 3$, while the density of unfriendly links is $1-\rho=L^{-} / L$.

It is useful to introduce the quantities $N_{k}^{+}$as follows: for each friendly link, count the number of triads of type $\Delta_{k}$ that are attached to this link. Then $N_{k}^{+}$is the average number of such triads over all friendly links. This number is

$N_{k}^{+}=\frac{(3-k) N_{k}}{L^{+}}$.

The factor $(3-k) N_{k}$ accounts for the fact that each of the $N_{k}$ triads of type $\Delta_{k}$ is attached to $3-k$ friendly links; dividing by $L^{+}$then gives the average number of such triads. Analogously, we introduce $N_{k}^{-}=k N_{k} / L^{-}$. Since the total number of triads attached to any given link equals $N-2$, the corresponding triad densities are (Fig. 3)

$n_{k}^{+}=\frac{N_{k}^{+}}{N-2}=\frac{(3-k) n_{k}}{3 n_{0}+2 n_{1}+n_{2}}$

$n_{k}^{-}=\frac{N_{k}^{-}}{N-2}=\frac{k n_{k}}{n_{1}+2 n_{2}+3 n_{3}}$.

We now write rate equations that account for the changes in the triad densities in an update. We choose a triad at random; if it is imbalanced $\left(\triangle_{1}\right.$ or $\left.\Delta_{3}\right)$ we change one of its links as shown in Fig. 2. Let $\pi^{+}$be the probability that a link changes from friendly to unfriendly in an update event, and vice versa for $\pi^{-}$. A friendly link changes to unfriendly with probability $1-p$ when $\triangle_{1} \rightarrow \Delta_{2}$, while an unfriendly link changes to friendly with probability $p$ if $\triangle_{1} \rightarrow \triangle_{0}$ and with probability 1 if $\triangle_{3} \rightarrow \triangle_{2}$. Consequently

$\pi^{+}=(1-p) n_{1} \quad \pi^{-}=p n_{1}+n_{3}$.

In the special case of $p=1 / 3$, each link of an imbalanced triad is flipped equiprobably. Since each update changes $N-2$ triads, 


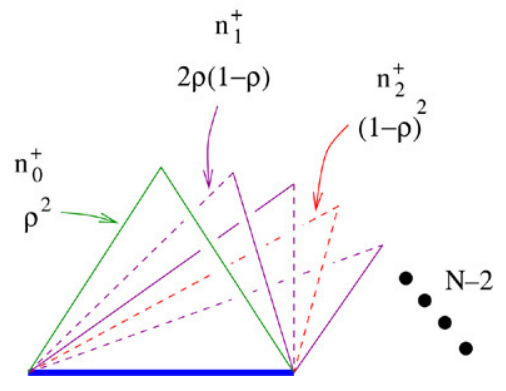

Fig. 3. Illustration of the different types of triads $(N-2$ in total) that are attached to a positive link (heavy line). Also shown are the stationary-state probabilities for each triad when the friendly link density is $\rho$. Full and dashed lines represent friendly and unfriendly relations, respectively.

and we define one time step as $L$ update events. Then the rate equations for the triad densities have the size-independent form

$\dot{n}_{0}=\pi^{-} n_{1}^{-}-\pi^{+} n_{0}^{+}$,

$\dot{n}_{1}=\pi^{+} n_{0}^{+}+\pi^{-} n_{2}^{-}-\pi^{-} n_{1}^{-}-\pi^{+} n_{1}^{+}$,

$\dot{n}_{2}=\pi^{+} n_{1}^{+}+\pi^{-} n_{3}^{-}-\pi^{-} n_{2}^{-}-\pi^{+} n_{2}^{+}$,

$\dot{n}_{3}=\pi^{+} n_{2}^{+}-\pi^{-} n_{3}^{-}$,

where the overdot denotes the time derivative.

Let us determine the stationary solution to these equations. Setting the left-hand sides of Eq. (5) to zero and also imposing $\pi^{+}=\pi^{-}$to ensure a fixed friendship density, we obtain $n_{0}^{+}=n_{1}^{-}, n_{1}^{+}=n_{2}^{-}, n_{2}^{+}=n_{3}^{-}$. Forming products such as $n_{0}^{+} n_{2}^{-}=n_{1}^{+} n_{1}^{-}$and using Eqs. (3a) and (3b), these relations are equivalent to

$3 n_{1} n_{3}=n_{2}^{2} \quad 3 n_{0} n_{2}=n_{1}^{2}$.

Furthermore, the stationarity condition, $\pi^{+}=\pi^{-}$, gives $n_{3}=(1-2 p) n_{1} \equiv n_{1} \delta / 3$, with $\delta=3(1-2 p)$. Using this result in the first of Eq. (6) gives $n_{2}=n_{1} \sqrt{\delta}$. Then the second of Eq. (6) gives $n_{0}=n_{1} /(3 \sqrt{\delta})$. We then write the normalization condition, $\sum n_{k}=1$, in terms of $n_{1}$ only to give

$\left(\frac{1}{3 \sqrt{\delta}}+1+\sqrt{\delta}+\frac{\delta}{3}\right) n_{1}=1$,

or

$n_{1}=\frac{3 \sqrt{\delta}}{(1+\sqrt{\delta})^{3}}$.

Finally, the stationary density of friendly links, $\rho=n_{0}+\frac{2}{3} n_{1}+$ $\frac{1}{3} n_{2}$ is

$\rho_{\infty}= \begin{cases}(1+\sqrt{\delta})^{-1} & p \leq 1 / 2 \\ 1 & p \geq 1 / 2 .\end{cases}$

Notice that the triad densities of each type become uncorrelated and are given by

$n_{j}=\left(\begin{array}{l}3 \\ j\end{array}\right) \rho_{\infty}^{3-j}\left(1-\rho_{\infty}\right)^{j}$.

As shown in Fig. 4, the stationary density of friendly links $\rho_{\infty}$ monotonically increases with $p$ for $0 \leq p \leq 1 / 2$ until utopia

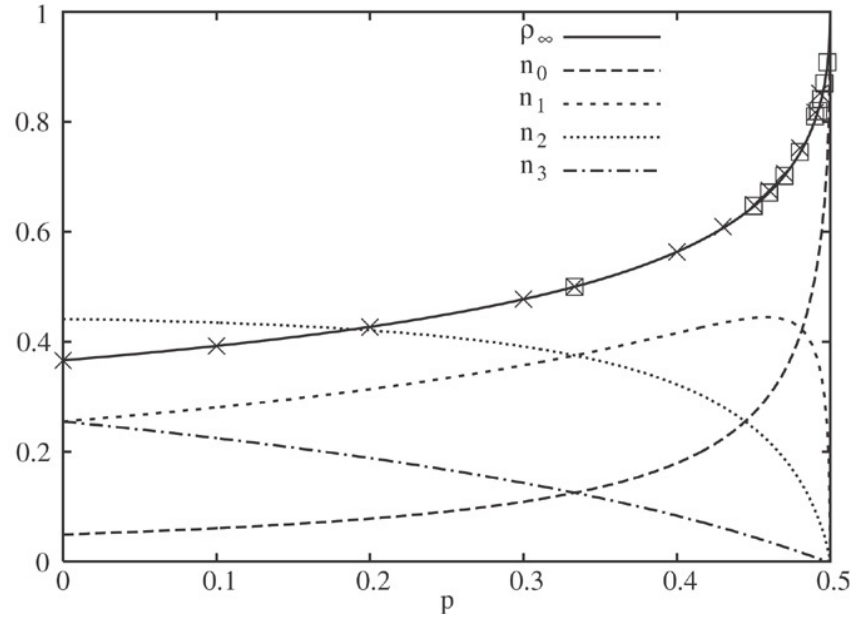

Fig. 4. The stationary densities $n_{k}(p)$ and the density of friendly links $\rho_{\infty}$ as a function of $p$. Simulation results for $\rho_{\infty}$ for $N=64$ (crosses) and 256 (boxes) are also shown. There is a similar degree of agreement between theory and simulation for $n_{k}$.

is reached. Near the phase transition, the density of unfriendly links $u \equiv 1-\rho_{\infty}$ vanishes as $\sqrt{3(1-2 p)}$.

\subsection{The evolving state}

A remarkable feature of the master equation (5) is that if the initial triad densities are given by Eq. (9) - uncorrelated densities - the densities will remain uncorrelated forever. In this case, it suffices to study the time evolution of the density of friendly links $\rho(t)$. We determine this time evolution directly by noting that $\rho(t)$ increases if $\Delta_{3} \rightarrow \Delta_{2}$ or $\Delta_{1} \rightarrow \Delta_{0}$, and decreases if $\Delta_{1} \rightarrow \Delta_{2}$. Since the respective probabilities for these processes are $1, p$, and $1-p$, we have

$\frac{\mathrm{d} \rho}{\mathrm{d} t}=3(2 p-1) \rho^{2}(1-\rho)+(1-\rho)^{3}$.

Solving this equation, the time dependence of the density of friendly links has the following behaviors:

$\rho(t)-\rho_{\infty} \sim \begin{cases}A \mathrm{e}^{-B t} & p<1 / 2 \\ -\frac{1-\rho_{0}}{\sqrt{1+2\left(1-\rho_{0}\right)^{2} t}} & p=1 / 2 \\ -C \mathrm{e}^{-3(2 p-1) t} & p>1 / 2,\end{cases}$

where $\rho_{0}$ and $\rho_{\infty}$ are the initial and final densities of friendly links, respectively, and $A, B$, and $C$ are constants that depend on $p$. Thus for $p \neq 1 / 2$ there is a quick approach to a final state. This state is frustrated for $p<1 / 2$ and is utopian for $p \geq 1 / 2$. For $p=1 / 2$ utopia is reached slowly - as a power-law in time.

\subsection{Fate of a finite society}

Abstractly, LTD represents a stochastic dynamics in a state space in which each network configuration is represented by a point in this space and a link to another point represents an allowed transition by the dynamics. Because balanced networks represent absorbing states of this dynamics, a finite network 


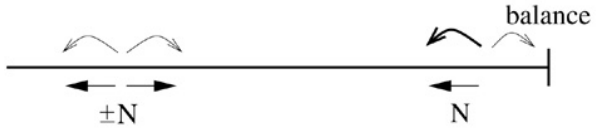

Fig. 5. Effective random walk picture for the approach to balance for $p<1 / 2$.

must ultimately fall into a balanced state for all $p$. We now estimate the size dependence of the time to reach a balanced state, $T_{N}$, for any value of $p$ by probabilistic arguments.

For $p<1 / 2$, we use the following random walk argument (Fig. 5): when a link is flipped on an imbalanced triad in an almost balanced network (nearly $N^{3} / 6$ balanced triads), then of the order of $N$ triads that contain this link will become imbalanced. Thus starting near balance, LTD is equivalent to a biased random walk in the state space of all network configurations, with the bias directed away from balance, and with the bias velocity $v$ proportional to $N$. Conversely, far from the balanced state, local triad dynamics is diffusive because the number of imbalanced triads changes by of the order of $\pm N$ equiprobably in a single update. The corresponding diffusion coefficient $D$ is then proportional to $N^{2}$. Since the total number of triads in a network of $N$ nodes is $N_{\triangle} \sim N^{3} / 6$, we therefore expect that the time $T_{N}$ to reach balance will scale as $T_{N} \sim$ $\mathrm{e}^{v N_{\Delta} / D} \sim \mathrm{e}^{N^{2}}$ [16]. The data in Fig. 7(a) is suggestive of faster than exponential growth of $T_{N}$ with $N$. A linear fit to the data for $\ln \ln T_{N}$ versus $\ln N$ gives a slope of 2.1, in excellent agreement with the prediction $T_{N} \sim \mathrm{e}^{N^{2}}$. Given that there are only 6 data points and the $N$ that we can simulate is small, this agreement is surprisingly good.

For $p>1 / 2$, we define the time to reach the balanced state by the naive criterion $u(t) \equiv 1-\rho(t)=N^{-2}$; that is, one unfriendly link remains. From Eq. (11), $T_{N}$ will then grow logarithmically with $N$. At $p=1 / 2$, using Eq. (11), the criterion $u(t)=N^{-2}$ now gives $T_{N} \sim N^{4}$. While simulations show that $T_{N}$ scales algebraically with $N$, the exponent is much smaller than 4 . The source of this smaller exponent is the fact that the number of unfriendly links fluctuates strongly about its mean value when there are few unfriendly links (see Fig. 6). To determine these fluctuations we write the number of unfriendly links in the canonical form [17]

$U(t)=L u(t)+\sqrt{L} \eta(t)$

where $u(t)$ is deterministic and $\eta(t)$ is a stochastic variable. Both $u$ and $\eta$ are size independent in the thermodynamic limit. A detailed argument [14] shows that $\sigma \equiv\left\langle\eta^{2}\right\rangle$ grows as $\sigma \sim \sqrt{t}$ as $t \rightarrow \infty$. Because of the finite-size fluctuations in $U$, the time to reach utopia $T_{N}$ is determined by the criterion that fluctuations in $U$ become of the same order as the average, viz.,

$\sqrt{L \sigma\left(T_{N}\right)} \sim \operatorname{Lu}\left(T_{N}\right)$.

Using $u(t) \sim 1 / \sqrt{t}$ from Eq. (11), $\sigma \sim \sqrt{t}$, and $L \sim N^{2}$, Eq. (13) becomes $N T_{N}^{1 / 4} \sim N^{2} T_{N}^{-1 / 2}$, from which $T_{N} \sim N^{4 / 3}$ follows.

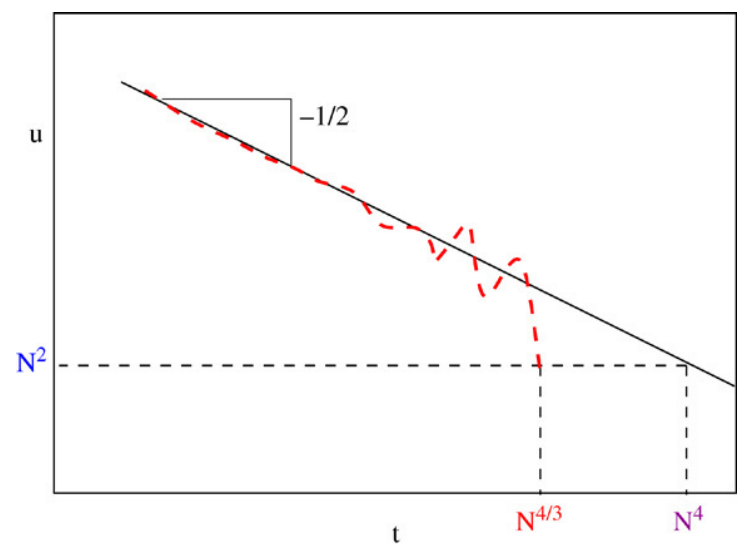

Fig. 6. Illustration of the rate equation solution for the unfriendly link density versus time on a double logarithmic scale and the influence of fluctuations on this solution.
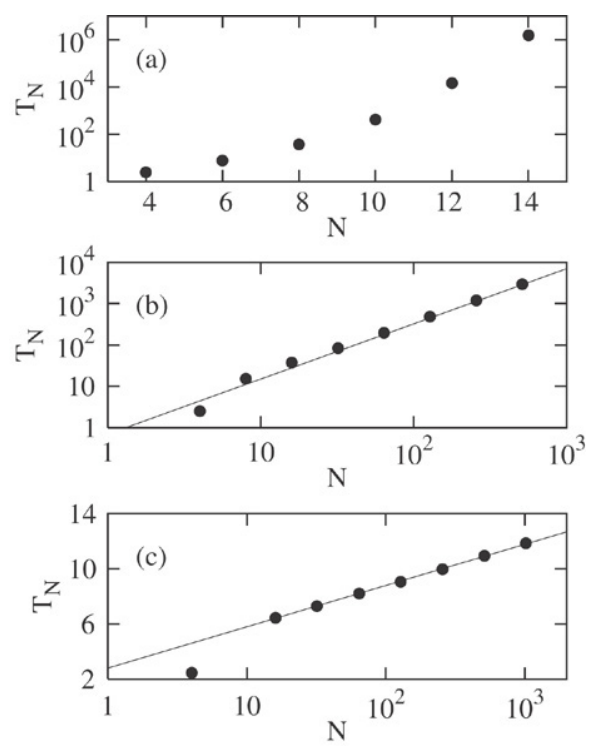

Fig. 7. Average time to reach balance as a function of $N$ for an initially antagonistic society $\left(\rho_{0}=0\right.$ ) for: (a) $p=1 / 3$; (b) $p=1 / 2$; (c) $p=3 / 4$. The line in (b) has slope $4 / 3$.

Summarizing our results, we have:

$T_{N} \propto \begin{cases}\mathrm{e}^{N^{2}} & p<1 / 2 \\ N^{4 / 3} & p=1 / 2 \\ (2 p-1)^{-1} \ln N & p>1 / 2 .\end{cases}$

These are in agreement with our simulation results shown in Fig. 7.

\section{Constrained triad dynamics}

In constrained triad dynamics (CTD), we first select an imbalanced triad at random and then select a random link in this triad. We change the sign of the link only if the total number of imbalanced triads decreases. If the total number of imbalanced triads is conserved in an update, then the update occurs with probability $1 / 2$. CTD can be viewed as the dynamics of a 


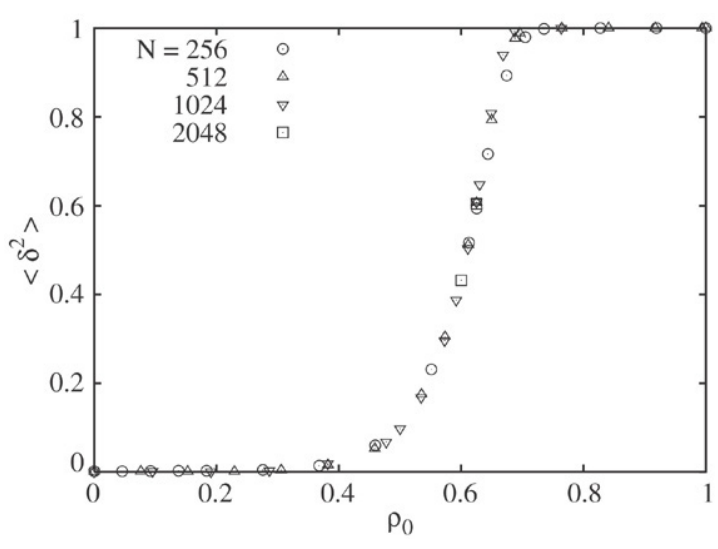

Fig. 8. Asymmetry of the final state as a function of the initial friendship density $\rho_{0}$ for several network sizes.

socially aware individual who considers her entire social circle before making any relationship change. Because of this global constraint, a network is quickly driven to a balanced state in a time that scales as $\ln N$.

A more interesting feature is the existence of a dynamical phase transition in the structure of the final state as a function of the initial friendly link density $\rho_{0}$ (Fig. 8). We quantify this structural change by the scaled difference in sizes of the two cliques in the final state, $\delta \equiv\left(C_{1}-C_{2}\right) / N$. For $\rho_{0}<0.4$ the cliques in the final state are nearly the same size and $\left\langle\delta^{2}\right\rangle \approx 0$. As $\rho_{0}$ increases toward $\rho_{0}^{*} \approx 2 / 3$, the size difference continuously increases and a sudden change occurs at $\rho_{0}^{*}$, beyond which the final state is utopia. Since $\left\langle\delta^{2}\right\rangle$ and the density of friendly links $\rho_{\infty}$ are related by $\left\langle\delta^{2}\right\rangle=2 \rho_{\infty}-1$ in a large balanced society, uncorrelated initial relations generically lead to $\rho_{\infty}>\rho_{0}$. Thus CTD tends to drive a network into a friendlier final state.

We now give a simple-minded argument that suggests that a large network undergoes a sudden change from $\rho_{\infty}=0$ (two equal size cliques) to $\rho_{\infty}=1$ (utopia) as a function of the initial friendly link density $\rho_{0}$. This qualitative approach predicts that this transition occurs at $\rho_{0}=1 / 2$. On the other hand, our numerical simulations show that the transition is located near $\rho_{0}^{*} \approx 2 / 3$ (Fig. 8).

Let us assume that a network remains uncorrelated during initial stages of evolution and under this assumption we determine the probabilities for a specific friendly link to flip. If the network is uncorrelated, the densities $\mathbf{n}^{+} \equiv$ $\left(n_{0}^{+}, n_{1}^{+}, n_{2}^{+}, n_{3}^{+}\right)$of triads that are attached to a friendly link are:

$\mathbf{n}^{+}=\left(\rho^{2}, 2 \rho(1-\rho),(1-\rho)^{2}, 0\right)$.

For a link to change from friendly to unfriendly, it is necessary that $n_{1}^{+}+n_{3}^{+}>n_{0}^{+}+n_{2}^{+}$. That is, this link is a member of more imbalanced triads than balanced triads. From Eq. (15), this condition is equivalent to $4 \rho(1-\rho)>1$, which never holds. Consequently, friendly links never flip. Similarly, the densities $\mathbf{n}^{-} \equiv\left(n_{0}^{-}, n_{1}^{-}, n_{2}^{-}, n_{3}^{-}\right)$of triads attached to an unfriendly link are:

$\mathbf{n}^{-}=\left(0, \rho^{2}, 2 \rho(1-\rho),(1-\rho)^{2}\right)$.
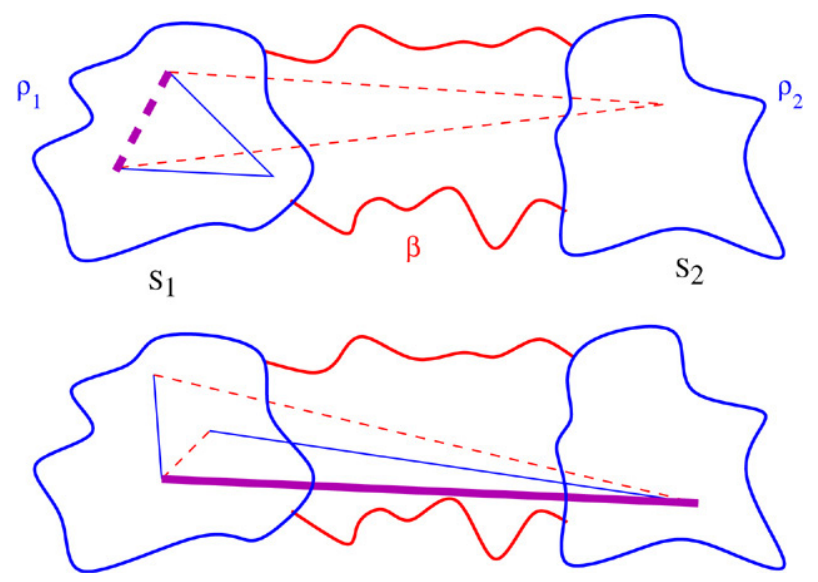

Fig. 9. Nascent cliques $S_{1}$ and $S_{2}$ (blobs at the extremities), with friendly link densities $\rho_{1}, \rho_{2} \gtrsim \frac{1}{2}$. The density of friendly links between cliques is $\beta \lesssim \frac{1}{2}$. Top: imbalanced triads that lead to an unfriendly link (thick dashed line) changing to a friendly link within one clique. Bottom: imbalanced triads that lead to a friendly link (thick solid line) changing to a unfriendly link between cliques.

To flip this unfriendly bond, we must have $n_{1}^{-}+n_{3}^{-}>n_{0}^{-}+n_{2}^{-}$, i.e., the bond is part of more imbalanced than balanced triads. This condition gives $1>4 \rho(1-\rho)$, which is valid when $\rho \neq$ $1 / 2$. Thus for a large uncorrelated network, only unfriendly links flip in CTD, except for $p=1 / 2$. Thus a network with $\rho_{0}>1 / 2$ should quickly evolve to utopia, while a network with $\rho_{0}<1 / 2$ should quickly approach a state where $\rho=1 / 2$.

Simulations indicate, however, that correlations in relationships occur when $\rho \approx 1 / 2$ and these ultimately lead to a bipolar society. We find that the precursor to this bipolar society is a state in which the network partitions itself by the dynamics into two subnetworks $S_{1}$ and $S_{2}$ of nearly equal sizes $C_{1}=\left|S_{1}\right|$ and $C_{2}=\left|S_{2}\right|$. Within each subnetwork, the density of friendly links $\rho_{1}$ and $\rho_{2}$ slightly exceeds $1 / 2$, while the density $\beta$ of friendly links between subnetworks is slightly less than $1 / 2$. This small fluctuation is amplified by CTD so that the final state is two nearly equal-size cliques.

To see how such evolution occurs, let us assume that relationships within each subnetwork and between subnetworks are homogeneous. Consider first the evolution within each clique (top panel of Fig. 9). For an unfriendly link in $S_{1}$, the densities of triads attached to this link are given by (16), with $\rho$ replaced by $\beta$ when the third vertex in the triad belongs to $S_{2}$, and by (16), with $\rho$ replaced by $\rho_{1}$ when the third vertex belongs to $S_{1}$. The requirement that a link can change from unfriendly to friendly by CTD now becomes

$C_{1}\left[1-4 \rho_{1}\left(1-\rho_{1}\right)\right]+C_{2}[1-4 \beta(1-\beta)]>0$,

which is always satisfied. Conversely, friendly links within each subnetwork can never change. As a result, negative intraclique links disappear and there is increased cohesiveness within cliques.

Consider now relations between cliques (bottom panel of Fig. 9). For a friendly link between the subnetworks, the triad densities attached to this link are

$\mathbf{n}_{j}^{+}=\left(\beta \rho_{j}, \beta\left(1-\rho_{j}\right)+\rho_{j}(1-\beta),(1-\beta)\left(1-\rho_{j}\right), 0\right)$ 


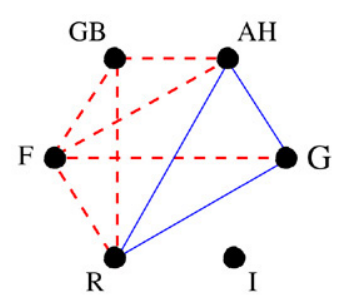

3 Emperor's league 1872-81

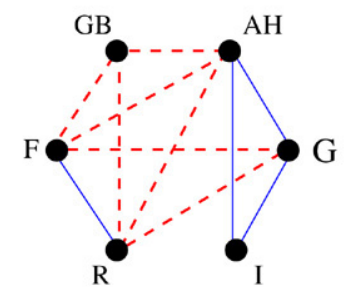

French-Russian Alliance1891 -94

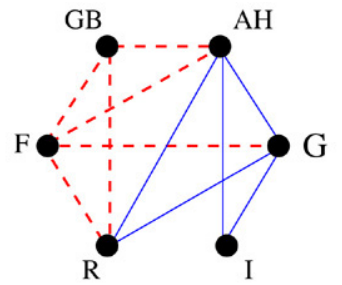

Triple Alliance 1882

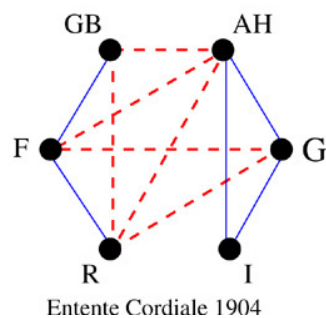

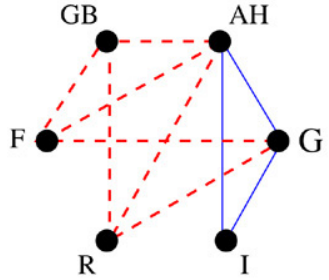

German-Russian Lapse 1890

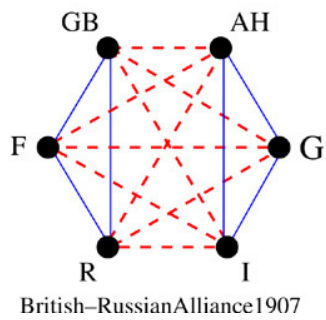

Fig. 10. Evolution of the major relationship changes between the protagonists of World War I from 1872-1907. Here GB = Great Britain, AH $=$ Austria-Hungary, $\mathrm{G}=$ Germany, $\mathrm{I}=$ Italy, $\mathrm{R}=$ Russia, and $\mathrm{F}=$ France.

when the third vertex belongs to $S_{j}$. Since

$$
\begin{aligned}
& \beta\left(1-\rho_{j}\right)+\rho_{j}(1-\beta)-\beta \rho_{j}-(1-\beta)\left(1-\rho_{j}\right) \\
& \quad=\left(2 \rho_{j}-1\right)(1-2 \beta),
\end{aligned}
$$

the change of a link from friendly to unfriendly is possible if

$\left[C_{1}\left(2 \rho_{1}-1\right)+C_{2}\left(2 \rho_{2}-1\right)\right](1-2 \beta)>0$.

Thus if the situation arises where $\rho_{1}>1 / 2, \rho_{2}>1 / 2$, and $\beta<1 / 2$, the network subsequently evolves to increase the density of intra-subnetwork friendly links and decrease the density of inter-subnetwork friendly links. This bias drives the network to a final bipolar state.

Finally, note that when $C_{1} \approx C_{2} \approx N / 2$, the number of ways, $\left(\begin{array}{c}N \\ C_{1}\end{array}\right)$, to partition the original network into the two nascent subnetworks $S_{1}$ and $S_{2}$, is maximal. Consequently, the partition in which $C_{1}=C_{2}$ has the highest likelihood of providing the initial link density fluctuation that ultimately leads to two nearly equal-size cliques, as observed in our simulations (Fig. 8). Although our argument fails to account for the precise location of the transition, the behavior of $\left\langle\delta^{2}\right\rangle$ in the two limiting cases of $\rho_{0} \rightarrow 0$ and $\rho_{0} \rightarrow 1$ is described correctly.

\section{Summary and discussion}

We presented a simple setting for social dynamics in which both friendly and unfriendly links exist in a network. These links evolve according to natural rules that reflect a social desire to avoid imbalanced triads. For local triad dynamics, a finite network falls into a socially-balanced state in a time that depends sensitively on the propensity $p$ for forming a friendly link in an update event. For an infinite network, a balanced state is never reached when $p<1 / 2$ and the system remains stationary. The density of unfriendly links gradually decreases and the network undergoes a dynamical phase transition to an absorbing, utopia state for $p \geq 1 / 2$.
For constrained triad dynamics, an arbitrary network is quickly driven to a balanced state. This rapid evolution results from the condition that the number of imbalanced triads cannot increase. There is also a phase transition from bipolarity to utopia as a function of the initial density of friendly links that arises because of small structural fluctuations that are then amplified by the dynamics.

It is interesting to consider the possible role of balance theory in international relations [18], with the evolution of the relations among the protagonists of World War I being a particularly compelling example (Fig. 10). A history starts with the Three Emperors' League (1872, and revived in 1881) that aligned Germany, Austria-Hungary, and Russia. The Triple Alliance was formed in 1882 that joined Germany, Austria-Hungary, and Italy into a bloc that continued until World War I. In 1890, a bipartite agreement between Germany and Russia lapsed and this ultimately led to the creation of a French-Russian alliance over the period 1891-94. Subsequently an Entente Cordiale between France and Great Britain was consummated in 1904, and then a British-Russian agreement in 1907, that then bound France, Great Britain, and Russia into the Triple Entente. While our account of these Byzantine maneuvers is incomplete (see Refs. [19] for more information), and Fig. 10 does not show all relations and thus the extent of network imbalance during the intermediate stages, the basic point is that these relationship changes gradually led to a reorganization of the relations between European nations into a socially balanced state. Thus while social balance is a natural outcome, it is not necessarily a good one!

We close with some potentially interesting open theoretical questions. First, it is natural to consider more general interactions. One can easily imagine ternary relationships of friendly + , unfriendly - , or indifferent 0 . Another possibility is continuous-valued interaction strengths. What is the number of cliques and number of communities as a function of network size and the density of indifferent relationships? Another direction, already considered by Davis [10], is a more Machiavellian society in which triads with three unfriendly 
relations are acceptable - that is "an enemy of my enemy may still be my enemy". This more relaxed definition for imbalanced triads may lead to interesting dynamical behavior that will be worthwhile to explore. Finally, what happens if relations are not symmetric, that is, $s_{i j} \neq s_{j i}$ ? How does one define balance or some other notion of social stability with asymmetric interactions?

\section{Note added in proof}

After this work was completed, a recent publication [20] has generalized our approach to dynamics on larger cycles and to incompletely connected networks.

\section{Acknowledgements}

TA gratefully acknowledges financial support from the Swiss National Science Foundation under the fellowship 8220067591. SR acknowledges financial support from NSF grant DMR0535503.

\section{References}

[1] F. Heider, Psychol. Rev. 51 (1944) 358-374;

F. Heider, J. Psychol. 21 (1946) 107-112;

F. Heider, The Psychology of Interpersonal Relations, John Wiley \& Sons, New York, 1958.

[2] K. Lewin, Field Theory in Social Science, Harper, New York, 1951.

[3] T.M. Newcomb, The Acquaintance Process, Holt, Rinehart \& Winston, New York, 1961
[4] S. Wasserman, K. Faust, Social Network Analysis: Methods and Applications, Cambridge University Press, New York, 1994.

[5] D. Cartwright, F. Harary, Psychol. Rev. 63 (1956) 277-293.

[6] T.M. Newcomb, Soc. Psychol. Quart. 42 (1979) 299-506.

[7] F. Harary, R.Z. Norman, D. Cartwright, Structural Models: An Introduction to the Theory of Directed Graphs, John Wiley \& Sons, New York, 1965.

[8] R.K. Leik, B.F. Meeker, Mathematical Sociology, Prentice-Hall, Englewood Cliffs, NJ, 1975.

[9] P. Bonacich, Introduction to mathematical sociology. http://www.sscnet. ucla.edu/soc/faculty/bonacich.

[10] J.A. Davis, Hum. Relat. 20 (1967) 181-187.

[11] N.P. Hummon, T.J. Fararo, J. Math. Sociol. 20 (1995) 145-159.

[12] P. Doreian, D. Krackhard, J. Math. Sociol. 25 (2001) 43-67.

[13] N.P. Hummon, P. Doreian, Soc. Networks 25 (2003) 17-49.

[14] More details of our approach are given in T. Antal, P.L. Krapivsky, S. Redner, Phys. Rev. E 72 (2005) 036121

[15] A study of a similar spirit to ours is given in K. Kulakowski, P. Gawronski, P. Gronek, Internat. J. Modern. Phys. C 16 (2005) 707;

P. Gawronski, P. Gronek, K. Kulakowski, Acta Phys. Polon. B 36 (2005) 2549.

[16] We use the fact that the first-passage time to an absorbing point in a finite one-dimensional interval of length $L$ with a bias away from the absorbing point is of the order of $\mathrm{e}^{v L / D}$. See S. Redner, A Guide to First-Passage Processes, Cambridge University Press, New York, 2001.

[17] N.G. Van Kampen, Stochastic Processes in Physics and Chemistry, NorthHolland, Amsterdam, 2003.

[18] See e.g. M. Moore, Eur. J. Soc. Psychol. 9 (1979) 323-326.

[19] W.L. Langer, European Alliances and Alignments 1871-1890, 2nd ed., Knopf, New York, 1950;

B.R. Schmitt, Triple Alliance and Triple Entente, Holt, Rinehart, and Winston, New York, 1934.

[20] F. Radicchi, D. Vilone, S. Yoon, H. Meyer-Ortmanns, Physics/0608031 